\title{
Probabilistic Modeling Confirms Delayed, But Non-Deficient \\ Locomotor Development in Low Birth Weight Piglets
}

keywords: locomotion, kinematics, probabilistic modeling, Fourier series, piglets, low birth weight, development

Falk Mielke, Chris Van Ginneken and Peter Aerts

2022-02-03

\section{Contents}

1 Abstract 


\section{Abstract}

In the study of vertebrate locomotion, kinematic measures of gait, dynamic posture, and coordination often change when comparing subjects of different body mass, size, and age. Is it, conversely, possible to infer subject characteristics from the kinematic measures? For this study, piglets (Sus domesticus) were filmed from lateral perspective during their first ten hours of life, an age at which body mass and size have major consequences for survival. We then apply deep learning methods for point digitization (DeepLabCut), calculate joint angle profiles, and apply an information-preserving transfromation (Fourier series) to retrieve a multivariate kinematic data set. We train a probabilistic model to predict subject characteristics from kinematics. The model infers subject characteristics accurately for strides from piglets of normal birth weight (i.e. the category it was trained on), but surprisingly predicts the body mass and size of low birth weight piglets (which were left out of training, out-of-sample prediction) to be "normal". The discrepancies between prediction and observation confirm that dynamic posture and coordination are unaffected by lower birth weight and lower size, which is evidence that low birth weight does not imply locomotor deficits. However, the age of some (but not all) low birth weight individuals is underestimated, supporting the hypothesis that these piglets experience a delay in locomotor maturation. (216 words) 


\section{Introduction}

Vertebrate locomotion is a complex phenomenon. The kinematic and dynamic measurements obtainable in experiments represent the collective output of interacting variables of an ensemble of subsystems: the musculoskeletal apparatus, energy supply, metabolism, and multiple levels of neuro-motor control (Nishikawa et al., 2007). All these subsystems are potentially affected by characteristics of the animal (e.g. age) and by external circumstances (e.g. friction) in different, non-trivial ways. Conversely, studying alterations in locomotor patterns under controlled experimental conditions holds diagnostic potential (e.g. Figueiredo et al., 2018). In well-studied species, it is even possible to identify individuals from kinematics (e.g. Patua et al., 2021).

Yet the complexity of the biological system is a major challenge to locomotor research. Experiments might record two animals of different weight, walking at different speeds, and producing two distinct kinematic patterns (e.g. one tending to bend the knee and ankle joints, the other emphasizing bending of knee and hip). Additionally, even for identical external conditions and in a single individual, there is intrinsic variation in the process, owing to the fact that "successive movements [...] never exactly repeat themselves" (Bernstein, 1935). Which of the many "input factors" is responsible for the difference in ensemble output, i.e. kinematics? This analysis question is common, and the solution is not novel. Multivariate models are capable of handling complex situations, given sufficient data. However, the high dimensionality of kinematic data sets, the multi-parameter, multi-level (hierarchical) covariate situations, and the high digitization workload have often been a limiting factor in the particular case of vertebrate locomotion (Jackson et al., 2016; Michelini et al., 2020; Seethapathi et al., 2019).

Several recent technological advances have enabled researchers to tackle scientific questions on locomotion in a more efficient way. Firstly, the past few years have brought huge leaps in terms of computer vision, deep learning, and thereby semi-automatic video digitization methods (Corcoran et al., 2021; Jackson et al., 2016; Karashchuk et al., 2021; Mathis et al., 2020; Mielke et al., 2020). These tools typically require a manually digitized subset of the data as the "training set" for a neural network, which is then able to digitize further videos in high through-put, hopefully with reasonable accuracy. A second field of technological advance are probabilistic models, which build on an elegant technical implementation of Bayesian theory (Markov Chain Monte Carlo / MCMC sampling, cf. Gelman et al., 2020; McElreath, 2018; van de Schoot et al., 2021). Such models can naturally incorporate hierarchical parameter interrelations 
and intrinsic variability. The main reason for this is that probabilistic models work on data distributions, and their outcome are distributions and "effect likelihoods", rather than point estimates. This can be informative on an intrinsically varying process such as locomotion (Mielke et al., 2018). These technical advances are gradually gaining more recognition in the field and are validly advancing to be the standard in kinematic analysis.

Piglets are a well-studied model system in which scientific interest joins the economic interest of commercial breeding. These animals have been subject to a variety of locomotor studies, including paradigms to test the effects of breed (Mirkiani et al., 2021), birth weight (Vanden Hole et al., 2018a, 2021, 2017), surface friction (von Wachenfelt et al., 2008), welfare (Guesgen and Bench, 2017), various pathologies (Abell et al., 2014; Benasson et al., 2020; LaVallee et al., 2020), and more ( $c f$. Netukova et al., 2021). Of particular interest has been the occurrence of a subset of individuals which are born with lower weight (LBW, low birth weight) than their "normal" (NBW) littermates. There are multiple standards to classify these birth weight categories, using absolute mass, litter quantile criteria, or asymmetry of body proportions (Amdi et al., 2013; D'Inca et al., 2011; Feldpausch et al., 2019; Quiniou et al., 2002; Roehe and Kalm, 2000; Van Tichelen et al., 2021; Wang et al., 2016). A possible cause of low birth weight is intra-uterine growth restriction, and LBW phenotype seems often, but not always, to correlate with low vitality and a reduced chance of survival (Baxter et al., 2008; Hales et al., 2013; Muns et al., 2013). On the other hand, locomotor maturation after birth is quick (Vanden Hole et al., 2021, 2017). These latter studies from our group raised the hypothesis that the apparent difference in LBW and NBW individuals can be attributed to delayed development. However, they measured spatiotemporal gait variables (e.g. stride frequency and distance, speed, duty factor), which are collective variables of the actual kinematics ( $c f$. Newell and Liu, 2021). This strategy has the advantage that it requires only five landmarks (four limbs, one reference) to be digitized, which can be a crucial trade-off to handle large data sets, yet it cannot capture information on intra-limb coordination (i.e. the relative timing of segmental movements within a limb; as opposed to inter-limb coordination, i.e. the relative timing of the cyling of the different limbs). Using the semi-automatic digitization techniques mentioned above, one can extend the analysis of gait variables to quantities of intra-limb coordination with manageable effort. At first instance, those additional quantities are "raw" joint angle temporal profiles. By applying a Fourier Series Decomposition (FSD; cf. Mielke et al., 2019), the joint angle 
profiles can be transformed to meaningful quantities such as dynamic posture (mean joint angle and effective range of motion), and coordination sensu stricto (relative phase and residual kinematics). An advantage of this transformation procedure is that it is reversible because all mathematical information is retained in the process (which is not the case when using collective variables alone), which means that joint angle profiles can be reconstructed for any observed or hypothetical point in parameter space. In this study, a conventional, 2D kinematics data set is extracted with the aid of deep learning tools from lateral videos of walking piglets. By applying multivariate analysis and FSD, we separate spatiotemporal gait variables, dynamic posture, and coordination, and model their relation to subject characteristics (mass, size, age, and birth weight category). All parameters are submitted to an inclusive, probabilistic model. We tackle the question of whether low birth weight is an indication of delayed development, and attempt to quantify the delay with an inverse modeling strategy as follows. Intuitively, and conventionally, joint kinematics are considered the output of the locomotor system. Therefore, conventional statistical models consider them on the "outcome" side; on the "input" side, the effects of birth weight, age, speed, or other parameters on the outcome are quantified. Herein, we use a different approach, and invert the model. We construct a probabilistic computer model which describes "age" and other subject characteristics as a function of all available kinematic parameters. The rationale is similar to that in subject recognition tasks (e.g. Patua et al., 2021): given a certain kinematic profile, can we infer characteristics of the subject which produced it? We split our data set into birth weight classes, and train the model on only the strides from NBW observations. We then use the model and compute out-of-sample prediction of all LBW observations.

Our hypothesis is that, if LBW were at the same stage of postnatal locomotor development as their NBW siblings, then the prediction should accurately infer the age of the LBW animals. Conversely, if the LBW piglets are delayed in development, the model would underestimate their age. Thus, by applying this inverse modeling strategy and comparing the computer-predicted age to the actual age of the LBW piglets, we can falsify or quantify a hypothesized delay in locomotor development. 


\section{Materials And Methods}

\subsection{Data Acquisition}

Recordings were done at a local farm in Belgium during several trips in October and November 2017. Farrowing was monitored to select Topigs x PIC piglets for another experiment (Ayuso et al., 2021). Piglets from selected litters were weighed at birth and numbered with non-toxic skin markers. Low birth weight (LBW) was classified by birth weight quantile (lowest $10 \%$ of each litter) and by a maximum mass of $800 \mathrm{~g}$ (D'Inca et al., 2011; Litten et al., 2003; Van Tichelen et al., 2021; Wang et al., 2016); all other piglets are assigned the NBW category. Shortly afterwards $(\approx 1-10 h)$, piglets were briefly taken from their pen and brought to a separate room for video recording (see below). Animals were recorded in pairs (as in Mielke et al., 2018), which drastically reduced anxiety and increased their motivation to cooperate. A few animals were recorded repeatedly, usually with a changing partner. Animals were ear-tagged and followed up: recording was repeated at approximately 4 and 10 days of age. That data was part of the digitization procedure, but excluded from the present analysis (see below). The subject characteristics documented for analysis are birth weight (continuous and categories "LBW"/"NBW" for "low" or "normal" birth weight), mass at recording, age (i.e. hours since farrowing) at recording, sex, and size. The size of the animal was approximated by a Principal Component Analysis (PCA) of digitization landmark distances along all segments ("size PCA", only first PC used, $93 \%$ of variability). Size and mass are expected to correlate, yet deviations would indicate animals of particularly slender or rotund habitus. All procedures followed ethical regulations and guidelines, and were approved by the Ethical Committee for Animal Testing of the University of Antwerp, Belgium (ECD 2015-26).

The recording room contained an elevated runway $(150 \times 50 \mathrm{~cm})$, covered with rubber mat to increase friction, and visible through a transparent frontal shield. Color videos were recorded (camera model: GC-PX100BE, JVC, Japan) at a temporal sampling rate of 50 frames per second and a spatial resolution of $1920 \times 1080$ pixels (later cropped to $500 \mathrm{px}$ height), from a distance at which the field of view would exactly capture the entire runway. A chess board at the back wall enabled spatial calibration. Video surveillance was permanent during the presence of the animals and stopped only in between recording sessions. Animals were able to move freely on the enclosed platform. To stimulate lateral locomotion, the two animals were repeatedly placed on opposite ends of the runway. Gentle tickling on the back and grunting vocalization of the researcher were other successful strategies to induce targeted locomotion 
lateral to the camera. After the recordings the piglets were returned to their litter and remained with the sow.

\subsection{Digitization}

We used the software DeepLabCut (DLC, Mathis et al., 2018) for digitization of all video material, in combination with custom made point tracking software (Mielke et al., 2020) to generate a training set. In total, our dataset contained 180 videos (more than 11 hours, 169 animals) of video. Our goal was to prepare a general DLC network which is capable of automatically tracking piglets at multiple ages, and which can be shared and re-used for subsequent research questions. This is why the full data set was used for digitization and for the calculation of some derived measures (size PCA). However, the analysis focus of this study (see below) was only a subset of the data, namely 58 animals of the youngest age class. The video processing workflow, applied to the full data set, was as follows. To get a balanced training set, one representative stride of each of the animals was selected, and the video was cut, cropped to runway height, and optionally mirrored horizontally so that movement would always be rightwards. All videos were concatenated and submitted to the DLC training set generation. DLC was set to select 2552 frames from these videos, which were tracked in an external software and re-imported for training (80\% training fraction). Seventeen landmarks (i.e. points of interest or "key-points"; usually joint centers) were digitized, representing all body parts visible on the lateral perspective (head: snout, eye, ear; back line: withers, croup, tail base; forelimb: scapula, shoulder, elbow, wrist, metacarpal, fore hoof; hindlimb: hip, knee, ankle, metatarsal, hind hoof). We selected a "resnet 152" network architecture and trained for 540, 672 iterations (16 days of computer workload). The network was then applied to digitize the full data set twice: once in default direction and once horizontally mirrored, because training set was always rightward movement.

Naturally, the trained network would only extract potentially useful landmark traces for episodes which resembled the training set, i.e. in episodes with a piglet moving perpendicular to the image axis, in lateral aspect and rightward direction. We automatically extracted 2597 of these by filtering for high digitization "likelihood" provided by DLC, low noise (i.e. steady landmark movement) and consistent, plausible landmark distances. We further applied an automatic algorithm to find footfalls and label stride cycles in the candidate episodes (4730 cycles). This procedure involved a start-end-matching 
optimization (using Procrustes superimposition) to ensure that strides were indeed cyclical. To further assess digitization quality, gait variables were automatically extracted. Definition of these variables was chosen to simplify the automatic procedure, as follows. Stride distance, frequency, and speed are trivial measures of the animal movement. Duty factor is available for fore- and hindlimb, and measures the fraction of time in which the respective hoof is in ground contact. Clearance is approximated by quantifying the ratio of flexion of each limb (one minus the quotient of minimum and maximum absolute hip-toe-distance during the stride). Head and torso angle are the stride-average angles of the snout-ear or withers-croup lines with respect to the static reference frame. Hindlimb phase measures the time between hind- and forehoof touchdown, divided by the stride cycle duration. Where applicable, gait variables were prepared for analysis (see below) by converting them to dimensionless values (Alexander and Jayes, 1983; Hof, 1996) using the cumulated distance of landmarks along the snout-to-tailbase line of the animal as reference, extracted as stride average from the digitized landmarks. Only strides with plausible values (i.e. those which lie within the theoretical distribution of each parameter; 1862 cycles) where processed. Manual inspection further boiled down the data set to 897 stride cycles (the others excluded for digitization errors, multi-animal confusion, non-walking gait, intermittent or sidewards locomotion, or incompleteness). Finally, 368 of the remaining strides from 58 animals were in the youngest age category $(<10 h)$ and thus selected for the present analysis, the data table is available online (see below).

\subsection{Data Processing}

The landmark data provided by DLC was further processed for analysis. Python code for the whole procedure is publicly available (https://git.sr.ht/ ${ }^{f a l k / p i g l e t \_f c a s, ~ P y t h o n ~ v e r s i o n ~} 3.10 .1$ at time of model calculation, https://www.python.org). First, joint angle profiles (i.e. joint angle values over time) were extracted for all relevant joints and for the total forelimb angle (croup-withers-hoof). Shoulder, elbow, wrist, hip, knee, and ankle were the six joints sufficiently well digitized and therefore considered relevant for analysis. We then applied Fourier Coefficient Affine Superimposition (FCAS, Mielke et al., 2019), a flexible procedure which subsumes the following steps. Joint angle profiles are cyclic, i.e. periodical, and can therefore be transformed to the frequency domain with a Fourier Series decomposition (8 harmonics). In the frequency domain, the affine components (mean, amplitude, 
phase) of a joint angle profile are easily accessible. The forelimb angle served as reference to temporally align all cycles in the data set (removal of phase differences between different cycles; forelimb angle was not used further). Then, mean and amplitude of the joint oscillations were isolated for all joint angles and are categorized as "dynamic posture" parameters. Mean joint angle is the temporal average, whereas amplitude is related to effective range of motion (eROM). The residual, i.e. relative phase differences captured by non-affine Fourier coefficients, is categorized as "coordination" sensu stricto (it measures the precise temporal succession of joint configurations). In our case, there are 96 variables of coordination (6 angles, 8 harmonics, real and imaginary) which are submitted to a PCA. Only the first 12 coordination components $(C C)$ are used for statistical analysis, capturing $76.4 \%$ of the variability in coordination. The number of components is chosen as a trade-off between information retention and limiting model complexity.

Information retention is generally a strength of this method. The neglected PCs and the residual not captured by 8 harmonics are the only information from kinematics of the given joints which are lost in this procedure. Apart from that, all information present in the raw joint angle profiles enters the analysis. Furthermore, all transformations are reversible, hence any analysis outcome can be translated back to kinematics with high accuracy, which bears a lot of herein unused potential, for example for interpolating unobserved subject states or for inferring kinematics of fossile species by phylogenetic and morphometric bracketing. Though we used a 2D dataset herein, the procedure could be applied equally well to angles measured from 3D coordinate data.

\subsection{Statistical Modeling}

To summarize, four categories of variables were used for analysis:

- subject characteristics: age, sex, mass, birth weight category, size

- spatiotemporal gait variables: distance, frequency, speed, clearance (fore-/hindlimb), duty factor (fore-/hindlimb), head angle, hindlimb phase

- dynamic posture: mean joint angles and eROM for six joints

- coordination: the residual after extraction of dynamic posture (see above)

Our research question is whether a probabilistic, linear model is able to predict subject characteristics 
(specifically: age, mass, and size, optionally logarithmized to comply with normality assumption) from raw kinematics (expressed as dynamic posture and coordination) and gait variables (collective variables). Given the common conception that kinematics are a complex output of an individual motor system, this might be considered an "inverse" modeling approach. The present analysis focused on three outcome variables (Fig. 1): mass $(k g)$, size (arb. units, from a PCA of marker distances), and age ( $h$, optionally log-transformed). Though these outcome variables were specific per individual and recording session, we analyzed them "per stride" (i.e. there were multiple strides with identical subject measures on the outcome side).

The model formula is:

$$
\theta \sim v_{1} \cdot \alpha+v_{s} \cdot \beta_{s}+\sum_{G} v_{g} \cdot \beta_{g}+\sum_{P} v_{p} \cdot \beta_{p}+\sum_{C} v_{c} \cdot \beta_{c}+\epsilon
$$

Herein, $\theta$ is either of the outcome subject characteristics, $\beta$ are slopes associated with the model parameters ( $s$ sex, $G$ gait variables, $P$ dynamic posture, $C$ coordination), $v$ are data vectors (e.g. $v_{1}$ is a vector of ones for the intercept $\alpha$, and $v_{s}$ is a boolean vector coding for subjects of 'sex $==$ male"), and $\epsilon$ is the model residual. Priors (i.e. a priori assigned distributions) for all slopes were Normal distributions with mean and standard deviation corresponding to the mean and two times standard deviation of the observed values of each parameter; logarithmic transform was applied where necessary. The observable ("likelihood") prior for $\theta$ was a Student's T distribution with a Gamma distributed $\nu$ (degrees of freedom; allows for wider-than-normal tails and robust regression), $\epsilon$ was modeled to be a Half Cauchy distribution. The model was implemented using the Python library "PyMC3" (version 3.11.4, Salvatier et al., 2016).

To re-emphasize, dynamic posture and coordination together capture almost all the kinematic information of the stride. Hence, we train the predictor model with all kinematics, gait variables, and sex. Birth weight category (LBW, NBW) is a filter parameter: we split our data set into LBW strides and two NBW subsets (training and validation). Training is performed by MCMC sampling ('sample' function in PyMC3), and a No U-Turn sampler was set to sample with 12 chains, each $2^{14}$ tuning and equally many sampling steps. All post-hoc model checks confirmed convergence (inspection of traces, bfmi $>0.94$ for all chains, Gelman-Rubin statistics $\approx 1$ for all parameters, sufficient effective sample size). Model comparison was performed, iteratively leaving out model parameters or replacing some by meaningful combinations (e.g. duty factor combined for fore- and hindlimb). However, because we follow and "all 

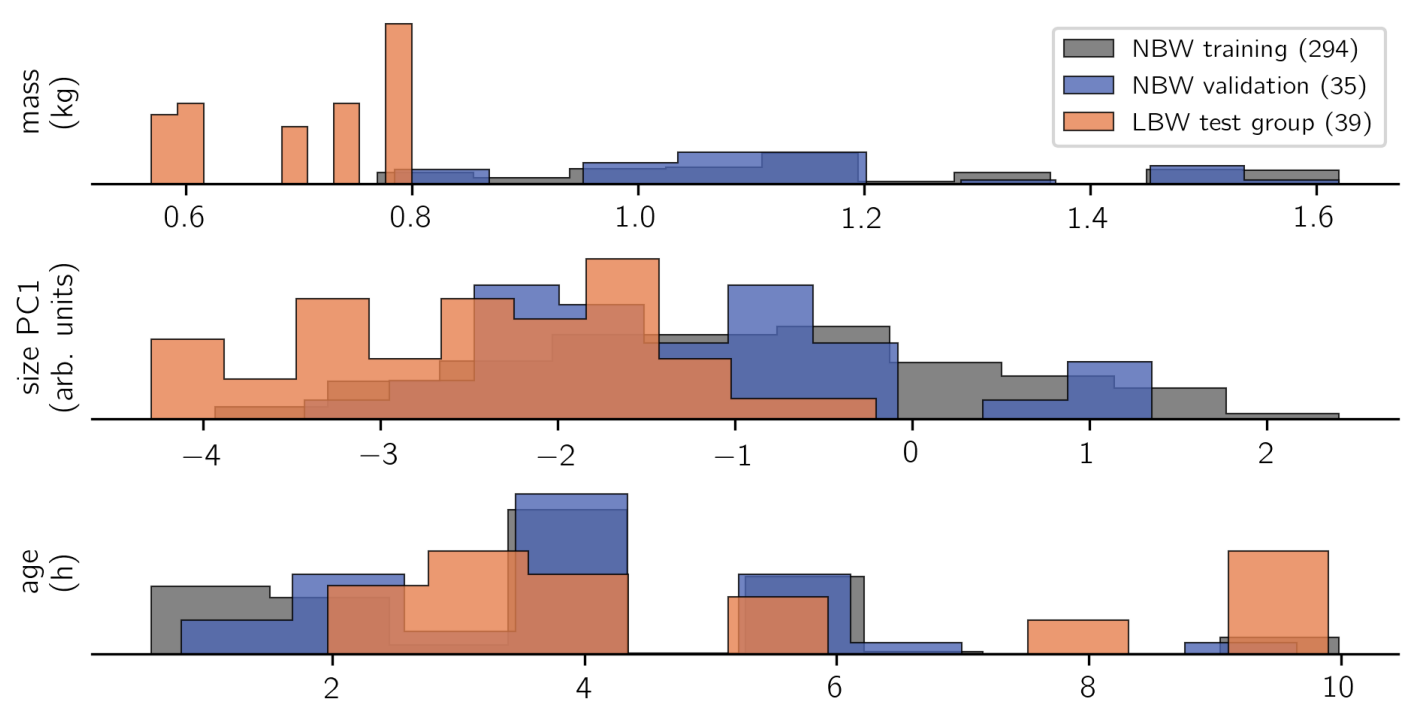

Figure 1: Histogram of observations. The LBW group measured the lowest body masses in the data set, which correlated with a lower body size. Recordings of all subsets happened opportunistically within the first ten life hours of the animals, repeated measurements were possible. Number of strides per class are indicated in brackets on the legend. Bar heights are scaled by sample size to show relative value distributions.

in" strategy, the results have little instructive value for model construction.

The data set of $N=368$ strides was split into three categories: (i) the NBW training set as reference with $N=294$ strides, (ii) the NBW validation set ( $N=35$ strides), which is a random subset of NBW strides, approximately equal in size to (iii) the LBW test set with $N=39$ strides.

The model was thus trained with a set of 294 NBW training strides (i). Predictions were then done per stride, for all observed strides (NBW training, NBW validation, and LBW test), iteratively using the 'pymc3.sample_posterior_predictive' function in PyMC3 after setting all the data arrays to the actual observed values for one given stride (using 'pymc3.set_data'). Due to a technical limitation, the number of predictions had to match the original sample size, and though repeated predictive sampling is possible, we found that number sufficient and would thus retrieve 294 mass, size, and age predictions for each stride in the data set.

All procedures, code, and data are available online in a git repository (https://git.sr.ht/ falk/ piglet_fcas). 


\section{Results}

The present analysis is centered around a linear model which is designed to infer mass, size, and age (subject characteristics) from an extensive set of kinematic parameters from $2 \mathrm{D}$ videos. The numbers provided by the model sampling are equally extensive, and will only be reported in brief. The key purpose of the model is posterior predictive sampling of the LBW strides which were left out of the model, and which are analyzed in detail below.

The outcome of MCMC sampling of a linear model are value distributions for slopes, which in our case indicated how certain kinematic parameters are associated with a change in mass, size, and age (supplementary material 7.1). None of the gait- or coordination parameters were correlated with differences in animal mass. However, mass was associated with changes in the dynamic posture of the hindlimb (hip, knee and ankle). For size, the model inferred associations with head angle, hindlimb duty factor and phase, and some coordination components (CC3, CC7), as well as changes in the foreand hindlimb posture and an effect of sex. Finally, age was associated with a change in forelimb clearance and duty factor (both increase with age), potential changes at the hip, ankle, and wrist, and several coordination components (CC6, CC9). Some eROM slope distributions for age were high in average magnitude, but variable (the "credible interval" contained zero). These model results provide detailed insight into parameter interrelations in the present data set and indicate which of the parameters are the relevent ones to infer a given subject attribute in predictive sampling.

Performing in-sample and out-of-sample prediction with the models trained on NBW strides elucidated if and how left-out strides differed from NBW model expectation (Fig. 2). Note that, to capture variance (i.e. uncertainty in the prediction), each stride was predicted repeatedly.

Out-of-sample predictions for the NBW validation set matched those of in-sample NBW prediction in terms of average values and standard deviation for all modeled outcome variables, which confirms that prediction of subject characteristics from kinematics is possible. In contrast, predictions for LBW strides did not match those of the NBW training set. Low birth weight animals were predicted on average $0.39 \mathrm{~kg}$ heavier than actual, and their size was overestimated $(+1.89$ units $)$. Both prediction faults matched the actual differences in magnitude ( $c f$. methods, Fig. 1). In contrast, the age predictions for the low birth weight subjects were bimodal: most ages were correctly inferred from stride-wise kinematics, but ages 

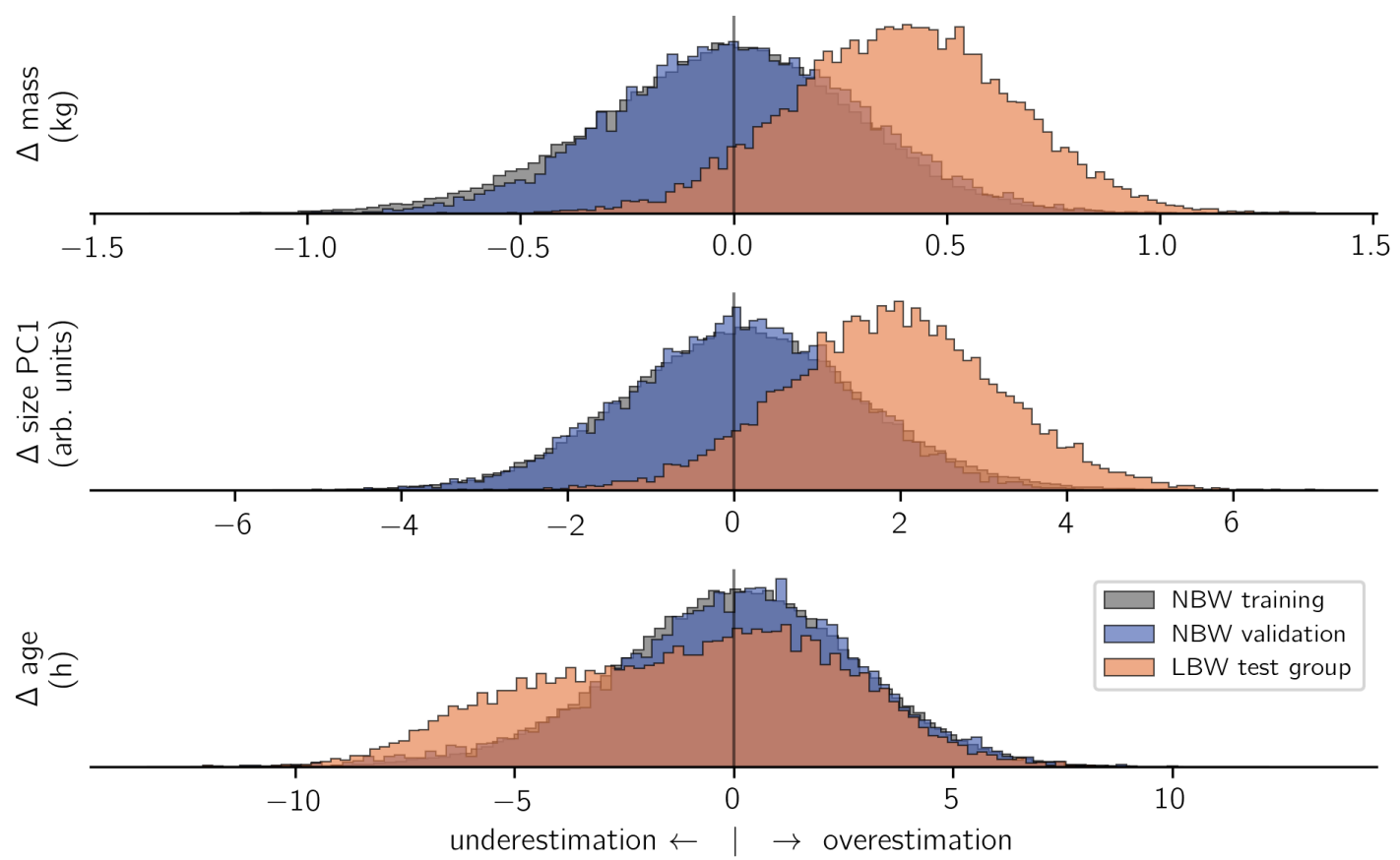

Figure 2: Model predictions. For all included subject characteristics, models which were trained on NBW strides correctly inferred the training data (gray) and values from the validation set (blue). In contrast, the same models wrongly inferred the characteristics of $L B W$ subjects (orange). The $x$-axes show the difference $(\Delta)$ between actual and predicted values per prediction. To facilitate comparison, histogram heights are again normalized per category. 
for some strides were underestimated. The underestimation of those strides quantified to less than five hours.

In summary, the NBW-trained model "guesses" the size and mass of the animals producing LBW strides to be "normal" (although they are not), which indicates that these defining features of LBW do not reflect in altered kinematics. However, age prediction is bimodal, i.e. some strides are classified as typical for animals of younger than actual age.

To find out whether the bimodality was related to certain individuals, or certain strides from different individuals, we grouped the predictions per stride or subject and calculated the chance of over- or underestimating age. Of the 8 low birth weight subjects who contributed 39 strides, 4 individuals were consistently underestimated (Tab. 1). Consistently means that more than $75 \%$ of all predictive samples were below actual age, and that the ages for a majority of strides were on average underestimated. The magnitude of underestimation was between two and five hours. Curiously, those were the individuals recorded at a slightly higher age ( $>5$ hours). Overestimation in the other four LBW individuals was also consistent, but less so (less extreme underestimation rate, mean $\Delta<2 h$ ). Standard deviation of the estimates did not vary across individuals or birth weight categories.

We conclude that underestimation of age is consistent over multiple strides of the same individual, and thus individual-specific. 
Table 1: Age predictions per LBW animal (compared to NBW average). $\Delta$ : "prediction - actual" difference. Underestimation is defined as $\Delta<0$, "count": per stride, "rate": per prediction. h: hours, std: standard deviation.

\begin{tabular}{|c|c|c|c|c|c|c|}
\hline piglet & $\begin{array}{l}\text { age } \\
\mathrm{h}\end{array}$ & strides & $\begin{array}{c}\text { underestimation } \\
\text { count }\end{array}$ & $\begin{array}{c}\text { underestimation } \\
\text { rate }\end{array}$ & $\begin{array}{c}\text { pred. mean } \Delta \\
\mathrm{h}\end{array}$ & $\begin{array}{c}\text { pred. std } \\
\text { h }\end{array}$ \\
\hline $\mathrm{b} 23$ & 2.0 & 6 & 0 & 0.26 & 1.47 & 2.00 \\
\hline b15 & 2.9 & 5 & 1 & 0.27 & 1.43 & 1.99 \\
\hline b76 & 3.1 & 4 & 1 & 0.44 & 0.28 & 1.96 \\
\hline b74 & 4.2 & 7 & 0 & 0.28 & 1.14 & 1.99 \\
\hline 1794.5 & 5.6 & 5 & 5 & 0.83 & -2.46 & 1.95 \\
\hline b58 & 7.8 & 3 & 3 & 0.95 & -3.41 & 2.01 \\
\hline b19v2 & 9.8 & 1 & 1 & 0.99 & -4.92 & 2.00 \\
\hline b56 & 9.9 & 8 & 8 & 0.98 & -4.75 & 2.02 \\
\hline$<$ all $\mathrm{NBW}>$ & $<3.8>$ & 329 & 146 & 0.49 & 0.02 & 1.97 \\
\hline
\end{tabular}




\section{Discussion}

Birth weight variability in piglets is considerable. The average birth weight of a new born piglet in our data set is just above a kilo, yet the span within a litter is typically above $800 \mathrm{~g}$. Size ranges are accordingly high. Intuitively, one would expect a pronounced difference in how these newborn animals move their more or less heavy bodies. The overarching question is whether low or critically low birth weight can be associated with a deficit in locomotion.

Previous studies reported behavioral, physiological, and other differences for piglets of low body mass which could affect locomotion (Alvarenga et al., 2013; Muns et al., 2013; Quiniou et al., 2002; Roelofs et al., 2019; Vanden Hole et al., 2018a,b). One might therefore anticipate differences in coordination, which is the immediate outcome of motor control. However, as our models have shown, neither mass, nor size were correctly retro-inferred from blindly providing kinematic parameters including coordination. Strides from LBW animals were estimated to come from an animal with a "normal" mass and size (Fig. 2). This confirms that low body mass or small size can not be causal for altered 2D kinematics, and it raises doubts whether there are any deficits in coordination and control. We observe LBW animals perfectly capable of normal posture, coordination, and overall stride results (collective variables). Note that this does not rule out problems of balance, stability, or endurance.

We do see differences for LBW compared to NBW recordings, nonetheless. First, a difference in sample size. There are much fewer valid LBW strides in our data set: only 39 of 368 observations are LBW. This could be interpreted as evidence for a lower capacity (despite equal potential) of LBW to produce normal locomotion. Yet there are proximal, trivial explanations: based on conventions, the $10 \%$ lower quantile of birth weights in a litter is considered LBW, and there is a hard cap of $800 \mathrm{~g}$. The resulting share is equal in our training set for video digitization, and in the final data set, because of pseudorandom, opportunistic sampling on-site (i.e. recording work was permanent, yet determined by farrowing and feeding of the subjects). The minority of LBW training videos might lead to an under-learning of those animals, reduced digitization quality and therefore an exclusion bias for "non-normal" individuals. Though it seems unlikely, we cannot rule out reduced locomotor capacity in LBWs, because the present data set is unsuited to count the occurrence of locomotor behavior. On the other hand, the strict stride

filtering criteria for "good" kinematics may have involuntarily filtered out deficient individuals. Our conclusion that low birth weight individuals are non-deficient is strictly tied to the definition of the low 
birth weight category, which is herein based on weight criteria and did not regard phenotypical indicators of intra-uterine growth restriction (which we did not record, cf. Amdi et al., 2013).

A second difference of LBW locomotion is that the age is underestimated for strides of some, but not all individuals. Prediction is consistent per individual, although no subject characteristics except sex entered the model ("blind" inference). This supports the hypothesis that locomotor development is sometimes delayed in LBWs. Delayed development does not necessarily corroborate the hypothesis of locomotor deficiency in LBW: we would expect truly deficient strides to be substantially different from the data trained to the model, making it "unpredictable" (i.e. higher variance of posterior samples). Instead, the predictions are consistent for repeated measures of an individual, without notable increase in variance. For the affected subjects, we can even quantify a delay of less than five hours, which could nevertheless be critical given the rapid maturation of locomotor behavior in this species (Vanden Hole et al., 2017) and the importance of postnatal competition (Litten et al., 2003).

Note however that the causality might be inverse. We measured age underestimation only in the case of the individuals which were recorded late within our sampling time frame (age $>5 h$ ). This is consistent with prior evidence that energy reserves are depleted after birth (normal locomotion) but not replenished after four hours in the case of LBW (Le Dividich et al., 2017; Vanden Hole et al., 2019). Assuming that this is the case, i.e. energy reserves are depleted, we would expect two effects: (i) the animal might succeed in the locomotor task less frequently (not quantified, see above), and (ii) the kinematics might be altered, which we observed. The present model was trained from NBW data and thereby tuned to kinematic development from animals with normal energy levels (therefore it can infer age from kinematics in NBW). That same model quantifies the potentially energy-deficient animals as younger. It might be that energy deficiency coincidentally causes effects which are exactly opposite to the changes that piglet kinematics undergo in normal development. However, the more likely explanation is an actual delay or a temporary halt in development. Failure of the LBW to compete in the first hours are sufficient to explain reduced intake (Amdi et al., 2013), the absolute size and mass difference alone might be crucial, and an immediate question which we cannot address with the present data set is whether and how (fast, likely) animals would recover from the delay. Alternatively, there might be a technical artifact in probabilistic sampling ("shrinkage", cf. Gelman et al., 2020) which caused the underestimation of "above average age" individuals. Yet this is an unlikely explanation, given that shrinkage would apply equally to NBWs, and 
inversely to the opposite, younger subjects. Finally, with only eight LBW individuals, we cannot exclude coincidence in which indivuduals are affected.

Neither of these technical explanations puts doubt on the clarity of the initial finding: a subset of the low birth weight individuals produced locomotor behavior which is quantitatively similar to that of younger individuals.

The findings discussed above are enabled by inverse-modeling subject characteristics as a function of kinematic parameters, using probabilistic models. The models do reveal various parameter associations, yet the top down (repeated) testing with the chosen model structure complicates drawing definite conclusions (e.g. we observed a sex effect on size, but opposite of what other studies have reported, Baxter et al., 2012, we conclude males in our study were just smaller by chance). Instead, the demonstrated strategy leverages the potential of probabilistic models to perform out-of-sample prediction (via separation of the LBW test group). Non-probabilistic modeling could equally serve to predict values, but it cannot generate parameter distributions (as in the bimodal age predictions, Fig. 2). Our probabilistic models implicitly regard "non-significant" parameter slopes, which are usually neglected in standard hypothesis testing (such as the high magnitude, highly variable joint ROM quantities, which might nevertheless have relevance for age prediction in case they are correlated and add up). The data transformations and predictive modeling strategy we applied herein hold further potential for inferring kinematics, for example by morphometric bracketing of extinct taxa. For that purpose, one would train a model to infer dynamic posture and coordination from a given range of morphometrics, predict samples for specific morphometrics in that range, and convert the samples back to (3D) joint angle profiles which could be animated.

There is a conceptual hierarchy, but no clear causality, when modeling parameter dependencies in quantitative studies of locomotion. For example, animals might increase the hip angle (posture) and the temporal pattern in the joint angle profiles (coordination) to reach higher speeds (spatiotemporal gait variables), but the speed they reach might depend on age (subject parameter). But age might also influence the speed without changing dynamic posture or coordination, simply because the animal grows and increases strength. Age might also affect dynamic posture and coordination directly, for example if dimensionless speed stays constant but clearance changes with age. Reducing clearance can increase speed (less unnecessary hoof lifting) or reduce it (higher duty factor), which can be distinguished by the 
other parameters. This complex interrelation of spatiotemporal quantities complicates the intuitive modeling strategy, which involves using subject characteristics as a factor to predict a multivariate block of gait variables and kinematics. In our experience, model residuals in such models are high, multiple testing can yield putatively coincidental significances, cross-dependencies within the multivariate data set might be underestimated, and sample size requirements are high. And even under ideal circumstances: chances are that such models would yield some age effect, even with random data.

In contrast, the strategy applied herein is related to the question: "given the complete kinematic output of a behavior, can we infer subject characteristics of the animal producing it?" We used probabilistic models, which are able to capture intrinsic variability of the process, and addressed specific categorical questions (NBW/LBW differences) by out-of-sample predictions. We demonstrated that, in the first ten hours of piglet life, (1) kinematics seem to be indifferent to low body mass and size, and (2) locomotion of some LBW individuals could be explained by a delay in locomotor maturation which is initiated post partum. 


\section{Acknowledgements}

The authors would like to thank Miriam Ayuso for organizing and participating in the recording sessions, as well as Laura Buyssens, Georgios Petrellis, Gunther Vrolix, Charlotte Vanden Hole, Denise Vogel and all students who joined for help during recordings. Maja Mielke provided valuable comments on the manuscript text. 
bioRxiv preprint doi: https://doi.org/10.1101/2022.02.04.479126; this version posted February 7, 2022. The copyright holder for this preprint (which was not certified by peer review) is the author/funder, who has granted bioRxiv a license to display the preprint in perpetuity. It is made available under aCC-BY 4.0 International license.

\section{Supplements}

\subsection{Detailed Modeling Results}

Asterisk $\left(^{*}\right)$ indicates slopes for which the credible interval did not include zero. FL: forelimb, HL: hindlimb, diml.: dimensionless, d.s.: dimensionless stride, eROM: effective range of motion 


\begin{tabular}{|c|c|c|c|c|}
\hline & age (h) & age $(\log )$ & size PC1 & mass $(\mathrm{kg})$ \\
\hline intercept & +2.93 & +0.78 & +2.19 & +1.03 \\
\hline female $\rightarrow$ male & +0.13 & +0.03 & $-0.36 *$ & -0.04 \\
\hline log. FL clearance & $+1.22 *$ & $+0.37 *$ & -0.53 & -0.11 \\
\hline log. HL clearance & +0.26 & +0.11 & -0.09 & +0.01 \\
\hline FL duty factor & +1.35 & $+0.46 *$ & +0.02 & +0.03 \\
\hline HL duty factor & +0.76 & +0.29 & $+0.78 *$ & +0.11 \\
\hline d.s. distance & +0.30 & -0.07 & +0.42 & +0.21 \\
\hline d.s. frequency & +2.76 & +1.32 & +2.13 & +0.13 \\
\hline diml. speed & -0.69 & -0.48 & +0.11 & -0.06 \\
\hline head angle & +0.57 & +0.24 & $+1.46 *$ & +0.11 \\
\hline hindlimb phase & -0.75 & -0.13 & $-2.59 *$ & -0.17 \\
\hline mean hip angle & $+2.16^{*}$ & +0.52 & -1.05 & $-0.52 *$ \\
\hline hip eROM & +2.68 & +1.06 & -1.76 & $-0.58 *$ \\
\hline mean knee angle & +0.25 & -0.02 & $-1.67 *$ & -0.14 \\
\hline knee eROM & +0.33 & +0.54 & $-2.54 *$ & $-0.45 *$ \\
\hline mean ankle angle & -1.43 & -0.74 & $+1.88 *$ & $+0.28 *$ \\
\hline ankle eROM & -2.15 & $-1.02 *$ & $+3.27 *$ & $+0.58 *$ \\
\hline mean shoulder angle & +1.32 & +0.20 & +0.39 & +0.02 \\
\hline shoulder eROM & -0.48 & -0.19 & -0.97 & -0.08 \\
\hline mean elbow angle & -1.07 & -0.55 & $+2.73 *$ & $+0.24 *$ \\
\hline elbow eROM & +0.42 & -0.04 & $+2.00 *$ & -0.01 \\
\hline mean wrist angle & $-2.51 *$ & $-1.17 *$ & +0.89 & -0.05 \\
\hline wrist eROM & -0.09 & +0.29 & -0.31 & +0.28 \\
\hline $\mathrm{CC} 1$ & +0.43 & +0.24 & +0.30 & +0.01 \\
\hline $\mathrm{CC} 2$ & $-0.85 *$ & -0.26 & +0.17 & +0.09 \\
\hline $\mathrm{CC} 3$ & +0.37 & +0.06 & $-0.79 *$ & -0.06 \\
\hline $\mathrm{CC} 4$ & -1.02 & $-0.39 *$ & +0.00 & +0.02 \\
\hline CC5 & +0.84 & +0.29 & -0.30 & -0.16 \\
\hline CC6 & $-1.42 *$ & $-0.57 *$ & +0.39 & +0.13 \\
\hline $\mathrm{CC} 7$ & +0.23 & +0.20 & $-0.84 *$ & -0.10 \\
\hline $\mathrm{CC} 8$ & -0.05 & +0.07 & -0.82 & -0.09 \\
\hline CC9 & $-3.28 *$ & $-0.92 *$ & +0.14 & +0.03 \\
\hline CC10 & -0.38 & -0.06 & -0.57 & +0.04 \\
\hline CC11 & -0.64 & -0.23 & +0.24 & +0.10 \\
\hline $\mathrm{CC} 12$ & -1.29 & -0.46 & -0.08 & -0.07 \\
\hline$\epsilon$ & \pm 1.79 & \pm 0.56 & \pm 0.98 & \pm 0.20 \\
\hline
\end{tabular}




\section{References}

Abell, C. E., Johnson, A. K., Karriker, L. A., Rothschild, M. F., Hoff, S. J., Sun, G., Fitzgerald, R., and Stalder, K. J. (2014). Using classification trees to detect induced sow lameness with a transient model. Animal, 8(6):1000-1009. doi: $10.1017 / \mathrm{S} 1751731114000871$.

Alexander, R. M. and Jayes, A. S. (1983). A dynamic similarity hypothesis for the gaits of quadrupedal mammals. Journal of Zoology, 201(1):135-152. doi: 10.1111/j.1469-7998.1983.tb04266.x.

Alvarenga, A., Chiarini-Garcia, H., Cardeal, P., Moreira, L., Foxcroft, G., Fontes, D., and Almeida, F. (2013). Intra-uterine growth retardation affects birthweight and postnatal development in pigs, impairing muscle accretion, duodenal mucosa morphology and carcass traits. Reproduction, Fertility and Development, 25(2):387-395. doi: 10.1071/RD12021.

Amdi, C., Krogh, U., Flummer, C., Oksbjerg, N., Hansen, C. F., and Theil, P. K. (2013). Intrauterine growth restricted piglets defined by their head shape ingest insufficient amounts of colostrum. Journal of Animal Science, 91(12):56055613. doi: $10.2527 /$ jas.2013-6824.

Ayuso, M., Irwin, R., Walsh, C., Van Cruchten, S., and Van Ginneken, C. (2021). Low birth weight female piglets show altered intestinal development, gene expression, and epigenetic changes at key developmental loci. The FASEB Journal, 35(4):e21522. doi: 10.1096/fj.202002587R.

Baxter, E., Jarvis, S., D'Eath, R., Ross, D., Robson, S., Farish, M., Nevison, I., Lawrence, A., and Edwards, S. (2008). Investigating the behavioural and physiological indicators of neonatal survival in pigs. Theriogenology, 69(6):773-783. doi: $10.1016 /$ j.theriogenology.2007.12.007.

Baxter, E. M., Jarvis, S., Palarea-Albaladejo, J., and Edwards, S. A. (2012). The Weaker Sex? The Propensity for Male-Biased Piglet Mortality. PLOS ONE, 7(1):1-9. doi: 10.1371/journal.pone.0030318.

Benasson, I., Wagnac, E., Diotalevi, L., Moore, D., Mac-Thiong, J.-M., and Petit, Y. (2020). Gait analysis of a post induced traumatic spinal cord injury porcine model. In 2020 42nd Annual International Conference of the IEEE Engineering in Medicine Biology Society (EMBC), pages 3803-3806.

Bernstein, N. A. (1935). The Problem of the Interrelation of Co-Ordination and Localization. In Whiting, H., editor, Human Motor Actions: Bernstein Reassessed (1984), volume 17 of Advances in Psychology, chapter 2, pages 77-119. North-Holland.

Corcoran, A. J., Schirmacher, M. R., Black, E., and Hedrick, T. L. (2021). ThruTracker: Open-Source Software for 2-D and 3-D Animal Video Tracking. bioRxiv. doi: 10.1101/2021.05.12.443854.

D'Inca, R., Gras-Le Guen, C., Che, L., Sangild, P. T., and Le Huërou-Luron, I. (2011). Intrauterine growth restriction delays feeding-induced gut adaptation in term newborn pigs. Neonatology, 99(3):208-216. doi: 10.1159/000314919.

Feldpausch, J. A., Jourquin, J., Bergstrom, J. R., Bargen, J. L., Bokenkroger, C. D., Davis, D. L., Gonzalez, J. M., Nelssen, J. L., Puls, C. L., Trout, W. E., and Ritter, M. J. (2019). Birth weight threshold for identifying piglets at risk for preweaning mortality. Translational Animal Science, 3(2):633-640. doi: 10.1093/tas/txz076.

Figueiredo, J., Santos, C. P., and Moreno, J. C. (2018). Automatic recognition of gait patterns in human motor disorders using machine learning: A review. Medical Engineering $\&$ Physics, 53:1-12. doi: 10.1016/j.medengphy.2017.12.006. 
Gelman, A., Carlin, J., Stern, H., Dunson, D., Vehtari, A., and Rubin, D. (2020). Bayesian Data Analysis. 3rd edition. http://www. stat.columbia.edu/ gelman/book.

Guesgen, M. and Bench, C. (2017). What can kinematics tell us about the affective states of animals? Animal Welfare, 26:383-397. doi: 10.7120/09627286.26.4.383.

Hales, J., Moustsen, V. A., Nielsen, M. B. F., and Hansen, C. F. (2013). Individual physical characteristics of neonatal piglets affect preweaning survival of piglets born in a noncrated system. Journal of Animal Science, 91(10):4991-5003. doi: $10.2527 /$ jas.2012-5740.

Hof, A. L. (1996). Scaling gait data to body size. Gait \& Posture, 4(3):222-223. doi: 10.1016/0966-6362(95)01057-2.

Jackson, B. E., Evangelista, D. J., Ray, D. D., and Hedrick, T. L. (2016). 3D for the people: multi-camera motion capture in the field with consumer-grade cameras and open source software. Biology Open, 5(9):1334-1342. doi: 10.1242/bio.018713.

Karashchuk, P., Rupp, K. L., Dickinson, E. S., Walling-Bell, S., Sanders, E., Azim, E., Brunton, B. W., and Tuthill, J. C. (2021). Anipose: A toolkit for robust markerless 3D pose estimation. Cell Reports, 36(13):109730. doi: 10.1016/j.celrep.2021.109730.

LaVallee, K. T., Maus, T. P., Stock, J. D., Stalder, K. J., Karriker, L. A., Murthy, N. S., Kanwar, R., Beutler, A. S., and Unger, M. D. (2020). Quantitation of Gait and Stance Alterations Due to Monosodium Iodoacetate-induced Knee Osteoarthritis in Yucatan Swine. Comparative medicine, 70(3):248-257.

Le Dividich, J., Charneca, R., and Thomas, F. (2017). Relationship between birth order, birth weight, colostrum intake, acquisition of passive immunity and pre-weaning mortality of piglets. Spanish Journal of Agricultural Research, 15(2):e0603. doi: 10.5424/sjar/2017152-9921.

Litten, J., Drury, P., Corson, A., Lean, I., and Clarke, L. (2003). The influence of piglet birth weight on physical and behavioural development in early life. Neonatology, 84(4):311-318.

Mathis, A., Mamidanna, P., Cury, K. M., Abe, T., Murthy, V. N., Mathis, M. W., and Bethge, M. (2018). DeepLabCut: markerless pose estimation of user-defined body parts with deep learning. Nature neuroscience, 21(9):1281-1289.

Mathis, A., Schneider, S., Lauer, J., and Mathis, M. W. (2020). A Primer on Motion Capture with Deep Learning: Principles, Pitfalls, and Perspectives. Neuron, 108(1):44-65. doi: 10.1016/j.neuron.2020.09.017.

McElreath, R. (2018). Statistical rethinking: A Bayesian course with examples in $R$ and Stan. Chapman and Hall/CRC.

Michelini, A., Eshraghi, A., and Andrysek, J. (2020). Two-dimensional video gait analysis: A systematic review of reliability, validity, and best practice considerations. Prosthetics and Orthotics International, 44(4):245-262.

Mielke, F., Schunke, V., Wölfer, J., and Nyakatura, J. A. (2018). Motion analysis of non-model organisms using a hierarchical model: Influence of setup enclosure dimensions on gait parameters of Swinhoe's striped squirrels as a test case. Zoology, 129:35-44. doi: 10.1016/j.zool.2018.05.009.

Mielke, F., Van Ginneken, C., and Aerts, P. (2019). Quantifying intralimb coordination of terrestrial ungulates with Fourier coefficient affine superimposition. Zoological Journal of the Linnean Society, 189(3):1067-1083. doi: 10.1093/zoolinnean/zlz135. 
Mielke, M., Aerts, P., Van Ginneken, C., Van Wassenbergh, S., and Mielke, F. (2020). Progressive tracking: a novel procedure to facilitate manual digitization of videos. Biology Open, 9(11). doi: 10.1242/bio.055962.

Mirkiani, S., Roszko, D. A., O’Sullivan, C. L., Faridi, P., Hu, D. S., Fang, D., Everaert, D. G., Toossi, A., Robinson, K., and Mushahwar, V. K. (2021). Overground Gait Kinematics and Muscle Activation Patterns in the Yucatan Mini Pig. bioRxiv. doi: 10.1101/2021.10.19.465020.

Muns, R., Manzanilla, E. G., Sol, C., Manteca, X., and Gasa, J. (2013). Piglet behavior as a measure of vitality and its influence on piglet survival and growth during lactation. Journal of Animal Science, 91(4):1838-1843. doi: $10.2527 /$ jas.2012-5501.

Netukova, S., Duspivova, T., Tesar, J., Bejtic, M., Baxa, M., Ellederova, Z., Szabo, Z., and Krupicka, R. (2021). Instrumented pig gait analysis: State-of-the-art. Journal of Veterinary Behavior, 45:51-59. doi: 10.1016/j.jveb.2021.06.006.

Newell, K. M. and Liu, Y.-T. (2021). Collective Variables and Task Constraints in Movement Coordination, Control and Skill. Journal of Motor Behavior, 53(6):770-796. doi: 10.1080/00222895.2020.1835799.

Nishikawa, K., Biewener, A. A., Aerts, P., Ahn, A. N., Chiel, H. J., Daley, M. A., Daniel, T. L., Full, R. J., Hale, M. E., Hedrick, T. L., Lappin, A. K., Nichols, T. R., Quinn, R. D., Satterlie, R. A., and Szymik, B. (2007). Neuromechanics: an integrative approach for understanding motor control. Integrative and Comparative Biology, 47(1):16-54. doi: $10.1093 / \mathrm{icb} / \mathrm{icm} 024$.

Patua, R., Muchhal, T., and Basu, S. (2021). Gait-Based Person Identification, Gender Classification, and Age Estimation: A Review. In Panigrahi, C. R., Pati, B., Mohapatra, P., Buyya, R., and Li, K.-C., editors, Progress in Advanced Computing and Intelligent Engineering, pages 62-74, Singapore. Springer Singapore.

Quiniou, N., Dagorn, J., and Gaudré, D. (2002). Variation of piglets' birth weight and consequences on subsequent performance. Livestock production science, 78(1):63-70. doi: 10.1016/S0301-6226(02)00181-1.

Roehe, R. and Kalm, E. (2000). Estimation of genetic and environmental risk factors associated with pre-weaning mortality in piglets using generalized linear mixed models. Animal Science, 70(2):227-240. doi: 10.1017/S1357729800054692.

Roelofs, S., Meijer, E., Baas, V., Dobrovolski, M., van der Staay, F. J., and Nordquist, R. E. (2019). Neurological functioning and fear responses in low and normal birth weight piglets. Applied Animal Behaviour Science, 220:104853. doi: 10.1016/j.applanim.2019.104853.

Salvatier, J., Wiecki, T. V., and Fonnesbeck, C. (2016). Probabilistic programming in Python using PyMC3. PeerJ Computer Science, 2:e55. doi: 10.7717/peerj-cs.55.

Seethapathi, N., Wang, S., Saluja, R., Blohm, G., and Kording, K. P. (2019). Movement science needs different pose tracking algorithms. arXiv, 1907.10226.

van de Schoot, R., Depaoli, S., King, R., Kramer, B., Märtens, K., Tadesse, M. G., Vannucci, M., Gelman, A., Veen, D., Willemsen, J., et al. (2021). Bayesian statistics and modelling. Nature Reviews Methods Primers, 1(1):1-26.

Van Tichelen, K., Prims, S., Ayuso, M., Van Kerschaver, C., Vandaele, M., Degroote, J., Van Cruchten, S., Michiels, J., and Van Ginneken, C. (2021). Handling Associated with Drenching Does Not Impact Survival and General Health of Low Birth Weight Piglets. Animals, 11(2). doi: 10.3390/ani11020404. 
Vanden Hole, C., Aerts, P., Prims, S., Ayuso, M., Van Cruchten, S., and Van Ginneken, C. (2018a). Does intrauterine crowding affect locomotor development? A comparative study of motor performance, neuromotor maturation and gait variability among piglets that differ in birth weight and vitality. PLOS ONE, 13(4):1-21. doi: 10.1371/journal.pone.0195961.

Vanden Hole, C., Ayuso, M., Aerts, P., Prims, S., Van Cruchten, S., and Van Ginneken, C. (2019). Glucose and glycogen levels in piglets that differ in birth weight and vitality. Heliyon, 5(9):e02510. doi: 10.1016/j.heliyon.2019.e02510.

Vanden Hole, C., Ayuso, M., Aerts, P., Van Cruchten, S., Thymann, T., Sangild, P. T., and Van Ginneken, C. (2021). Preterm Birth Affects Early Motor Development in Pigs. Frontiers in Pediatrics, 9:1003. doi: 10.3389/fped.2021.731877.

Vanden Hole, C., Cleuren, S., Van Ginneken, C., Prims, S., Ayuso, M., Van Cruchten, S., and Aerts, P. (2018b). How does intrauterine crowding affect locomotor performance in newborn pigs? A study of force generating capacity and muscle composition of the hind limb. PLOS ONE, 13(12):1-18. doi: 10.1371/journal.pone.0209233.

Vanden Hole, C., Goyens, J., Prims, S., Fransen, E., Ayuso Hernando, M., Van Cruchten, S., Aerts, P., and Van Ginneken, C. (2017). How innate is locomotion in precocial animals? A study on the early development of spatio-temporal gait variables and gait symmetry in piglets. Journal of Experimental Biology, 220(15):2706-2716. doi: 10.1242/jeb.157693.

von Wachenfelt, H., Pinzke, S., Nilsson, C., Olsson, O., and Ehlorsson, C.-J. (2008). Gait analysis of unprovoked pig gait on clean and fouled concrete surfaces. Biosystems Engineering, 101(3):376-382. doi: 10.1016/j.biosystemseng.2008.09.002.

Wang, J., Yang, M., Cao, M., Lin, Y., Che, L., Duraipandiyan, V., Al-Dhabi, N. A., Fang, Z., Xu, S., Feng, B., Liu, G., and Wu, D. (2016). Moderately increased energy intake during gestation improves body condition of primiparous sows, piglet growth performance, and milk fat and protein output. Livestock Science, 194:23-30. doi: 10.1016/j.livsci.2016.09.012. 